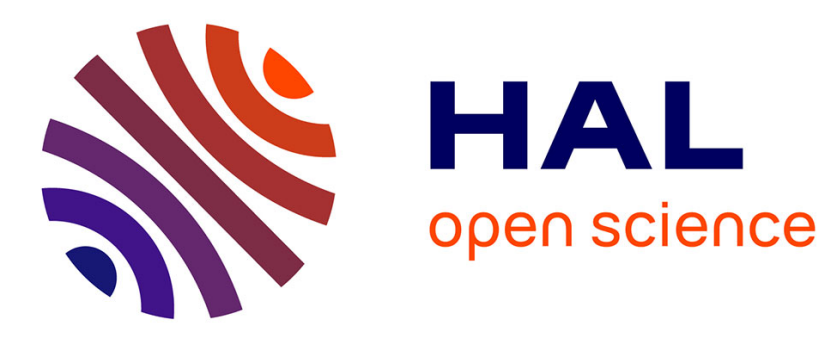

\title{
Loss of affineness in gels and melts
}

\author{
J. Bastide, J. Herz, François Boué
}

\section{- To cite this version:}

J. Bastide, J. Herz, François Boué. Loss of affineness in gels and melts. Journal de Physique, 1985, 46 (11), pp.1967-1979. 10.1051/jphys:0198500460110196700 . jpa-00210146

\section{HAL Id: jpa-00210146 https://hal.science/jpa-00210146}

Submitted on 1 Jan 1985

HAL is a multi-disciplinary open access archive for the deposit and dissemination of scientific research documents, whether they are published or not. The documents may come from teaching and research institutions in France or abroad, or from public or private research centers.
L'archive ouverte pluridisciplinaire HAL, est destinée au dépôt et à la diffusion de documents scientifiques de niveau recherche, publiés ou non, émanant des établissements d'enseignement et de recherche français ou étrangers, des laboratoires publics ou privés. 
Classification

Physics Abstracts

$46.30 \mathrm{~J}-61.12-61.40 \mathrm{~K}$

\title{
Loss of affineness in gels and melts
}

\author{
J. Bastide $\left({ }^{+}\right)$, J. Herz $\left({ }^{+}\right)$and F. Boué $\left({ }^{*}\right)$ \\ $\left({ }^{+}\right)$Centre de Recherche sur les Macromolécules, 6, rue Boussingault, 67083 Strasbourg Cedex, France \\ (*) Laboratoire Léon Brillouin, CEA Saclay, 91191 Gif/Yvette Cedex, France
}

(Reçu le 3 août 1984, révisé le 28 mai 1985, accepté le 26 juin 1985)

Résumé. - Nous discutons des mesures, par diffraction de neutrons aux petits angles, du facteur de forme d'une longue chaîne $\left(M>2 \times 10^{6}\right)$ dans deux milieux déformés, de déformation comparable :

(i) Un fondu étiré 4,6 fois (soit en direction perpendiculaire, une déformation $1 / \sqrt{4,6} \sim 0,46$ ). La longue chaîne, deutérée, est dissoute dans de longues chaînes hydrogénées.

(ii) Un gel, réticulé en solution, déformé par séchage (dégonflement de 10, soit dans une direction, une déformation de $\left.10^{-1 / 3} \sim 0,46\right)$. La longue chaîne deutérée est réticulée avec les autres chaînes.

Nous pensons qu'une comparaison directe des deux facteurs de forme est intéressante, pour les temps considérés (" plateau caoutchoutique ») : la chaîne est un chemin marqué obligé à passer, pour le gel, par de nombreux points de réticulation, pour le fondu, par de nombreux points d'enchevêtrements équivalents, dans ce domaine de temps, à des points de réticulation (les mailles sont comparables).

En plus de cette comparaison directe nous comparons aux deux modèles classiques de l'élasticité caoutchoutique (affine et fantôme), pour lesquels nous avons calculé le facteur de forme.

Malgré certaines différences les données pour les deux systèmes sont plus proches entre elles qu'elles ne le sont des modèles classiques : le désaccord́ avec ceux-ci suggère une perte d'affinité à des échelles plus grandes que la maille.

Abstract. - We discuss measurements by mean of small angle neutron scattering of the form factor of a long chain $\left(M>2 \times 10^{6}\right)$ in two comparably deformed materials :

(i) A melt, stretched 4.6 times (leading, in a perpendicular direction, to a deformation $1 / \sqrt{4.6} \sim 0.46$ ). The long chain, deuterated, is dissolved in the matrix non-deuterated chains.

(ii) A gel, crosslinked in solution, deformed by drying (deswelling of 10, leading in one direction to a deformation of $10^{-1 / 3} \sim 0.46$ too). The long chain was crosslinked with the other non-deuterated chains.

We believe that a direct comparison of the two form factors is interesting for the times here involved (« rubbery plateau ") : the chain is a labelled path which is obliged, for the gel, to pass across numerous crosslinks and, for the melt, to pass through numerous entanglement points (meshes are comparable in the two cases).

In addition to this direct comparison, we compare the two classical models of rubber elasticity (affine and phantom), for which we have calculated the form factor.

In spite of some differences, data for the two systems agree more between them than they do with classical models : the disagreement with those suggests a loss of affineness at larger scales than the mesh.

\section{Introduction.}

The purpose of this paper is to set up a scientific background and to use preliminary data for comparing two media.

- A gel is synthesized by crosslinking a semi-dilute solution $(\varphi=0.1, \varphi$ polymer volume fraction) of large polystyrene chains in a $\theta$ solvent (cyclopentane, see below). The deformation considered is a deswelling from 10 times in volume to the dry state.
- A melt is constituted by large polystyrene chains in bulk. The deformation process is the following : first the sample is stretched rapidly in the molten state, above the glass transition temperature; then, it is kept in a constant shape and after a time $t$ quenched in the glassy state, which freezes the relaxation.

Though different, both systems have a common feature : a state of elastic deformation, i.e. one that is highly recoverable when the constraints are released. For the first system, the constraint is to impose the 
density of bulk chains upon the sample. Swelling the sample at the initial concentration in the same solvent would release the constraint. The deswelling is isotropic : in any direction, the deformation ratio is therefore $(1 / 10)^{1 / 3}=0.464$. For the second system, the constraint is to impose a new length $L=4.6 L_{0}$, with $L_{0}$ being the initial length. Since the deformation is performed at a constant volume, the deformation ratio in a perpendicular direction is $1 /(4.6)^{1 / 2}=0.466$. In this paper, we consider only small values of time in the case of the melt

$$
t \ll T_{\text {ter }}
$$

$T_{\text {ter }}$ being the maximum time of relaxation of the system. The strong dependence of $T_{\text {ter }}$ upon the molecular weight $M$ of the chains $\left(T_{\text {ter }} \propto M^{3.4}\right)$ allows condition (1a) to be verified if the chains are large (here $M \sim 2.5 \times 10^{6}$ ). This leads to a quasitotal recovering when the constraint is released : that is, if after the time $t$, the sample is unclamped instead of quenched, its length returns nearly to $L_{0}$. An additional condition

$$
\left\{\tau_{\mathrm{tr}}^{\mathrm{i}}\right\} \ll t
$$

is satisfied, where $\left\{\tau_{\mathrm{tr}}^{\mathrm{i}}\right\}$ represents the short time spectrum. (In other words, the rheologists would call the range of times corresponding to bulk (1a) and (1b) the "rubbery plateau regime" [1].) The short time spectrum also exists for permanent networks : since the duration of the deswelling, $t^{\prime}$, is very large, condition (1b) is fulfilled for our dried gel. On the other hand, condition (1a) for $t^{\prime}$ is also verified, since the maximum time of relaxation of the gel is infinite.

Thus, a close relationship exists between the two systems in spite of their apparent differences. Moreover, a parallel can be drawn between these systems in the sense that both are labelled, a requirement for small angle neutron scattering experiments. In the case of the melt, we mixed deuterated chains with undeuterated chains : this allowed us to measure the form factor of the deuterated chain $[2,3]$ modified by the deformation. In the case of the gel, instead of labelling the elementary mesh as was done previously $[4,5]$, we labelled a path in the network : i.e., the same deuterated chains as used for the melt were mixed into a solution of undeuterated chains. After crosslinking, a gel in which these chains were statistically linked to the network structure at a large number of points was formed (see below). Following the same treatment as for the melt, we could measure the form factor of this labelled path in the deformed state.

In addition to making a direct comparison between the two form factors, we were able to test the theories usually applied to those kinds of systems.

An essential feature of the rubberlike elasticity is the loss of affineness of the deformation when the scale is decreased. The deformation of these materials appears to be classically homothetic to the deformation of the sample down to the scale of the micron.
On the other hand, at the very local scale, the monomer unit is not deformed. Consequently, the deformation « needs » to be progressively lost at a semi-local scale. The textbook model of the conformation of one chain linked by its extremities to fixed points but free to rearrange between them shows such a loss of affineness. Thus, a first satisfactory explanation of the rubber elasticity was obtained by identifying a network with a collection of such chains, i.e. connected to fixed crosslinks the positions of which are displaced affinely in the macroscopic strain [6]. This consideration of the crosslinks as such particular points is less im sortant in a second model called the "phantom network ». An additional freedom is given here : the crosslinks are assumed to fluctuate freely around affinely transformed mean positions [7].

These models were originally developed for permanent networks and for slow deformation (i.e. those with condition (1a) for the time $t^{\prime}$ characteristic of the deformation). But they should also apply to polymer melts under certain conditions. These conditions must fulfil both (1a) and (1b). Then the melts of long chains are described as temporary networks, the temporary crosslinks being attributed in modern theories to the entanglement points. In the range of times considered here, this network of entanglements cannot be destroyed by Brownian motion, so the melt should be considered comparable to a permanent rubber and be described by the same theories.

First we want to establish whether both systems undergo the same loss of affineness and, second, to test classical theoretical descriptions of process. Small angle neutron scattering conveniently furnishes information on pair correlations at any scale along the labelled chain. As the labelled objects are large, we can probe the deformation on a large range of distances. These distances cover the whole semi-local range, which is the pertinent region in which to observe the loss of affineness. The theoretical description of this region will finally be tested by comparing the experimental data to the theoretical form factors which we have evaluated numerically.

\section{Experimental.}

2.1 SYNTHESIS OF HIGH MOLECULAR WEIGHT POLYSTYRENE SAMPLES. - Polystyrene and deuterated polystyrene samples were prepared at low temperature $\left(-70^{\circ} \mathrm{C}\right)$ in tetrahydrofurane solution by anionic polymerization. The synthesis was carried out in a glass reactor under a slight argon pressure. Anhydrous tetrahydrofurane (THF) was obtained by distillation from a sodium benzophenone solution and kept under argon. The monomers were distilled on sodiumwire. A monofunctional carbanionic compound in THF solution, phenylethyl potassium, was used as the polymerization initiator.

The anionic synthesis of polymers in the high molecular weight range (above $10^{6} \mathrm{~g} \cdot \mathrm{mol}^{-1}$ ) requires 
extreme experimental conditions excluding the presence of even minute traces of proton-donating impurities capable of « killing " the growing polymer chain-ends. Therefore the solvent is first introduced into the reactor and the impurities are deactivated by the addition of some drops of the initiator solution until persistence of the characteristic red colour of the initiator-carbanion. About $10 \%$ in volume monomer is then added, and the very small traces of impurities still present in the styrene are neutralized by the addition of a few drops of a diphenylmethyl potassium solution in THF until persistence of a pole red colour. The latter organometallic compound is able to react with the impurities but does not initiate the polymerization reaction of styrene. Polymerization starts only after the very small amount of initiator solution required to obtain samples in the high molecular weight range is added.

After the reaction, the carbonionic chain ends are protonated by the addition of methanol. The polymer, obtained in an almost quantitative yield, is then precipitated in methanol, washed and dried.

Polymers in a molecular weight range between $10^{6}$ and $4 \times 10^{6} \mathrm{~g} . \mathrm{mol}^{-1}$ were thus prepared. Their molecular weight distribution ranged between 1.3 and 1.8, and some of the samples were fractionated.

Deformed melt samples. - Strips of polystyrene of $2 \mathrm{~cm} \times 8 \mathrm{~cm} \times 1 \mathrm{~mm}$ were made from a mixture of deuterated chains dispersed in ordinary polystyrene chains of smaller molecular weight (see Sect. 3). The mixture was then cast and moulded [8-10]. The strips were stretched uniaxially, at a constant temperature of $T=140^{\circ} \mathrm{C}$ (well above the glass transition temperature of $100{ }^{\circ} \mathrm{C}$ ) in a silicone oil bath; the elongation ratio was 4.6 and the duration of elongation was $5 \mathrm{~s}$. The samples were left at constant length for a time $t$, then taken out of the bath and brought to room temperature (quenching). Some of the samples were first observed after a first relaxation $\left(t=t_{1}\right)$, then dipped again in the oil bath for a time $t_{2}$ : this is taken to be the equivalent of a total duration $t=t_{1}+t_{2}$. For the purpose of this paper we observed only the values of $t=10 \mathrm{~s}, 1 \mathrm{~min}, 10 \mathrm{~min}$, and $40 \mathrm{~min}$; we give data for two values of $t, 1 \mathrm{~min}$ and $10 \mathrm{~min}$. The elongation ratio was tested by marks on the samples by comparing the distance between the marks before and after the deformation relaxation. It was verified that the macroscopic deformation was such that

$$
\mathbf{r} \rightarrow E \cdot \mathbf{r}, \quad E=\left(\begin{array}{ccc}
\lambda & 0 & 0 \\
0 & 1 / \sqrt{\lambda} & 0 \\
0 & 0 & 1 / \sqrt{\lambda}
\end{array}\right) .
$$

In the same way we took care to observe a part of the stretched strip where no change of shape occurred during the second relaxation. The obtained samples, ready to be put into the neutron beam, were then kept at room temperature. Different features about the molecular weight will be discussed in section 3 .

Gel samples. - Gels were prepared from mixtures of $1 \%$ (in volume) of the same deuterated chains as used in the melt and $99 \%$ of a protonated matrix (see Table I) dissolved in cyclopentane at a polymer volume fraction of 0.1 . Cyclopentane is a $\theta$ solvent for polystyrene at $T=20^{\circ} \mathrm{C}$. At $T=25^{\circ} \mathrm{C}\left(\sim 20^{\circ} \mathrm{C}\right)$, the statistics of the chain are Gaussian at short scales (i.e. shorter than the size of the «thermal blob» [11]). On the other hand, since the concentration 0.1 corresponds to the semi-dilute regime, the statistics are Gaussian at large scales too (because intrachain correlations are screened by interchain interactions [12]). These polymer mixtures were radiation crosslinked by exposure to a gamma ray source (Co 60) at $25^{\circ} \mathrm{C}$. The description of the mechanism of the reaction will be published elsewhere [13]. It is, however, important to note that the solvent molecules on which radicals are initiated not only act as a diluant but also play an active role in the chemical linking of the chains. The practical consequences are the low doses of irradiation necessary to achieve crosslinking $(10 \%$ of that required

Table I. - Molecular weights of polymers (measurements of C. Strazielle, A. Lapp, C.R.M.).

\begin{tabular}{|c|c|c|c|c|}
\hline \multirow{2}{*}{ Samples } & \multirow{2}{*}{$\begin{array}{l}\text { Light scattering } \\
M_{\mathrm{w}}\end{array}$} & \multicolumn{3}{|c|}{ Gel permeation chromatography } \\
\hline & & $M_{\mathrm{w}} / M_{\mathrm{n}}$ & $M_{\mathrm{w}}$ & $M_{\text {peak }}$ \\
\hline $\begin{array}{l}\text { Labelled chain (in both gel \& } \\
\text { melt) }\end{array}$ & $2.9 \times 10^{6}$ & 1.33 & $2.6 \times 10^{6}$ & $2.3 \times 10^{6}$ \\
\hline Matrix for gel (S 572) & $7.2 \times 10^{5} \pm 5 \%$ & 1.15 & $7.6 \times 10^{5}$ & \\
\hline $\begin{array}{l}\text { Matrix for melts ( } \mathrm{S} 507 \text {, before } \\
\text { moulding) }\end{array}$ & & 1.17 & $1.535 \times 10^{6}$ & $1.527 \times 10^{6}$ \\
\hline $\begin{array}{l}\text { (after moulding \& annealing) } \\
\text { (GPC FB2 2/3/84) }\end{array}$ & & $\sim 1.6$ & $9.8 \times 10^{5}$ & $1.15 \times 10^{6}$ \\
\hline
\end{tabular}

$\left(^{*}\right)$ For deuterated chains, the real masses are given (i.e. $\propto m_{\mathrm{D}}=112$ instead of $m_{\mathrm{H}}=104$ for the mass of the monomer). 
to crosslink bulk polystyrene) and the very small rate of chain cutting. Depending on the time of irradiation, gels with various crosslinking densities were obtained and studied by SANS. In this paper we will focus on one sample, R54. It can be characterized by its swelling degree $Q=V / V_{\text {dry }}$ ( $V$ equals the volume of the swollen sample, $V_{\text {dry }}$ equals the volume of the dry sample), measured using an optical method [14], [39] by its elastic modulus $G$ (measured in uniaxial compression) in pure toluene, and by its cooperative diffusion constant $D_{\mathrm{c}}$, again in pure toluene, determined in Dr. Candau's laboratory by quasielastic light scattering spectroscopy analysis [15]. The obtained values

$$
\begin{aligned}
Q & =23 \pm 2 \\
D_{\mathrm{c}} & =9.5 \pm 0.5 \times 10^{-7} \mathrm{~cm}^{2} \mathrm{~s}^{-1} \\
G & =3.1 \times 10^{4} \text { dynes } \mathrm{cm}^{-2}
\end{aligned}
$$

are very comparable to those of calibrated gels synthesized by endlinking elementary chains with molecular weight $M_{\mathrm{w}}=35000$ [15]. Though the crosslinking is undoubtedly less regular for the present gel, we believe that the value $M \simeq 35000$ is a reasonable estimation of the average molecular weight of the network mesh. To study the deswelling mechanism, we dried the samples and cut them in the shape of small disks which were then polished; from the state of preparation where the chains are Gaussian, the deswelling is ten times in volume. An isotropic disk of the uncrosslinked mixture (melt) was prepared at the same time.

Neutron experiments. - The two measurements were performed at two different periods, once using the D11 spectrometer and the other the D17 spectrometer (both of ILL reactor, Grenoble), in a setting giving approximately the same $q$ characteristics $(q$ is the scattering vector). The detectors are bidimensional. Thus, in the case of stretched samples the scattering was anisotropic and cells of the same $q$ modulus regrouped by sectors of 20 (resp. 10) degrees for parallel (resp. perpendicular) direction to the elongation axis, giving $S\left(q_{\|}\right)$(resp. $S\left(q_{\perp}\right)$ ). For deswollen gels the scattering was isotropic (as was the deswelling), and the regrouping was annular.

In both cases the signal was subtracted from the incoherent part due to hydrogen nuclei and normalized by transmission thickness. The obtained quantity is called normalized coherent scattering and will be noted $S(q)$.

\section{Problems associated with the difference between the molecular weights of the labelled chains and the matrix.}

In an ideal experiment, the molecular weights of the deuterated and the non-deuterated chains should be equal. This would have the following two advantages :

- For the melt sample, the characteristic relaxa- tion times would be the same for labelled and matrix chains and fulfil equations (1a) and (1b) in the same way.

- For both gels and melts which belong to the case of uncompressible binary mixtures, it has been shown [3] that the normalized coherent scattered cross section $S(q)$ can be expressed, within the framework of a good approximation, as follows :

$$
1 / S(q)=1 /\left[\left(1-\phi_{\mathrm{D}}\right) N_{\mathrm{H}} P_{\mathrm{H}}(q)\right]+1 /\left[\phi_{\mathrm{D}} N_{\mathrm{D}} P_{\mathrm{D}}(q)\right]
$$

where $S_{\mathrm{H}}(q)=N_{\mathrm{H}} P_{\mathrm{H}}(q)$ and $S_{\mathrm{D}}(q)=N_{\mathrm{D}} P_{\mathrm{D}}(q)$ represent respectively the (intramolecular) form factor of the deuterated and undeuterated chains. $N_{\mathrm{H}}$ and $N_{\mathrm{D}}$ represent the polymerization indexes of these chains respectively. This relation can also be put in the form

$$
S(q) /\left(1-\phi_{\mathrm{D}}\right) \phi_{\mathrm{D}}=S_{\mathrm{D}}(q)\left(1 /\left[1-\phi_{\mathrm{D}}\left(1-S_{\mathrm{D}}(q) / S_{\mathrm{H}}(q)\right)\right]\right)
$$

and if $N_{\mathrm{H}}$ now equals $N_{\mathrm{D}}$, the equation reduces to

$$
S(q) /\left[\left(1-\phi_{\mathrm{D}}\right) \phi_{\mathrm{D}}\right]=S_{\mathrm{D}}(q)
$$

and this allows us to extract $S_{\mathrm{D}}(q)$ from one measurement.

Here $\mathrm{H}$ and $\mathrm{D}$ masses are different in both the melt and the gel before crosslinking. The precise values of the masses are in table I. For the gel before crosslinking, $M_{\mathrm{D}}=2.6 \times 10^{6}$ and $M_{\mathrm{H}}=7.6 \times 10^{5}$; for the melt, before annealing (see below), $M_{\mathrm{D}}=2.6 \times$ $10^{6}$ and $M_{\mathrm{H}}=1.6 \times 10^{6}$.

3.1 EXTRACTION OF THE FORM FACTOR. - We nOw use equation (3) with different $S_{\mathrm{H}}$ and $S_{\mathrm{D}}$. This would make necessary to do at least two experiments (for two values of $\phi_{\mathrm{D}}$ ) with exactly the same deformation history, which is difficult. Here we have just used equation (4) to obtain $S_{\mathrm{D}}(q)$; thus our result is spoiled by the form factor of the non-deuterated chains. The error $\varepsilon(q)$ that it produces is evaluated in appendix 1 . There we give the form factor for a chain of mass $8 \times 10^{5}$ deformed under same conditions (by drying a gel). At high $q$, the curves are rather close together; the error is maximum at the lowest $q$. For the gel, because $\phi_{\mathrm{D}}$ is small it will never be more than $1 \%$. For the melt, it will be $5 \%$ for $q=1$ to $2 \times 10^{-2} \AA^{-1}$, but less than $1 \%$ for $q=6$ to $8 \times 10^{-2} \AA^{-1}$.

\subsection{RelaXATION TIMES AND PlATEAU BEHAVIOUR ; -} The difference between $M_{\mathrm{H}}$ and $M_{\mathrm{D}}$ has no rheological effect for the gel since we are far above the gelation point (see Ref. [13]; $M_{\mathrm{H}} / M_{\text {mesh }}>20$ ). However it has an effect for the melt. As explained in the introduction we need to obey

$$
\tau_{\mathrm{tr}}^{\mathrm{i}} \ll t \ll T_{\text {ter }}
$$

often called the " rubbery plateau regime ». The lefthand inequality (i.e. Eq. (1b)) allows us to assume 
that only the terminal time is relevant - that is, the modulus is well described by a single exponential $G(t)=G_{\mathrm{N}}^{0} \exp \left(-t / T_{\text {ter }}\right)$. The right-hand inequality allows us to assume a very weak decay of $G(t):$ it appears close to a plateau in $\log G(t)-\log t$ representation. Estimation of the maximum time of the $\tau_{\mathrm{tr}}^{\mathrm{i}}$, $\tau_{\mathrm{tr}}^{\max }$ is done in references [1] and [8,9] as follows. The Rouse model (free chain in a viscous medium) is in agreement with the data for $t<\tau_{\mathrm{tr}}^{\max } \cdot \tau_{\mathrm{tr}}^{\max }$ appears equal to the maximum Rouse time of a chain of mass $M_{\mathrm{e}} \cdot M_{\mathrm{e}}$ is the molecular weight between entanglement as pulled from $G_{\mathrm{N}}^{0}=k_{\mathrm{B}} T / M_{\mathrm{e}}$, as detailed in reference [1]. In addition, all times depend on the temperature by the same prefactor (WLF superposition) [26]. We made various estimations of the different times in earlier $[8,9,30]$ papers. At a standard temperature of $117^{\circ} \mathrm{C}$, we gave

$$
\tau_{\mathrm{tr}}^{\max } \cong 10 \mathrm{~s}
$$

and we checked the value of terminal time for $M=$ $7.6 \times 10^{5}$ in different ways $[8,9,10,40]$ :

$$
T_{\text {ter }}\left(7.6 \times 10^{5}\right) \cong 5 \times 10^{5} \text { to } 5 \times 10^{6} \mathrm{~s}
$$

Taking a $M^{3}$ dependence (weaker than the actual $M^{3.4}$, we obtain

$$
T_{\text {ter }}\left(1.6 \times 10^{6}\right) \cong 4 \times 10^{6} \text { to } 4 \times 10^{7} \mathrm{~s}=T_{\text {termat }}
$$

for the matrix chain, and

$$
T_{\text {ter }}\left(2.6 \times 10^{6}\right) \cong 2 \times 10^{6} \text { to } 2 \times 10^{7} \mathrm{~s}=T_{\text {terlab }}
$$

for the labelled chain. Times $t$ used in the experiment are $60 \mathrm{~s}$ and $600 \mathrm{~s}$ at $T=140^{\circ} \mathrm{C}$, which gives $3 \times 10^{4}$ and $3 \times 10^{5} \mathrm{~s}$ at $117^{\circ} \mathrm{C}$ using WLF superposition coefficients [1]. Thus, condition (1b) is fulfilled $(3 \times$ $\left.10^{4} \mathrm{~s}, 3 \times 10^{5} \mathrm{~s} \gg 10 \mathrm{~s}\right)$, as is (1a) for $T_{\text {terlab }}$ as well as for $T_{\text {termat }}$.

3.3 EFFECT OF ANNEALING. - Before performing the step strain and relaxation experiment the melt sample must be perfectly relaxed. That is, after moulding (which induces strains) it must be annealed. The duration of this process is proportional to the maximum relaxation time. When both the matrix and the labelled chain are very large, the latter is very long, even at high temperature (e.g., $T_{\text {ter }}>10$ hours at $180^{\circ} \mathrm{C}$ for $\left.M=2.6 \times 10^{6}\right)$. At such temperatures another process becomes important : the chemical degradation. Therefore, in order to reduce the time of annealing, we chose a lower molecular weight (by a factor of 2) for the matrix. In spite of this choice, a final G.P.C. analysis of the samples after annealing revealed an increase in the polydispersity from 1.15 to 1.5 for the matrix chains (there is no information about the deuterated chains because they are in too low a concentration). We believe that this is not too substantial a modification. A possible check for the deuterated chain is that of the form factor for the isotropic case : it is close to the theoretical one for a monodisperse chain and also close to that of the same chain dissolved in the smaller matrix chains used for the gels, for which the melt wafer was annealed ten times less. Recent experiments to be reported soon allowed the degradation to be sensibly reduced.

\section{Measured form factors.}

4.1 DifFICULTIES IN QUANTIFYING THE DEFORMATION AT THE SCALES EXPLORED. - How can the rate of deformation at intramolecular distance from our $S(q)$ data be appreciated ? Can we summarize this set of values by a simple number which would characterize the deformation at the length scale which we are exploring ? The answer to the second question is " no" for this experiment. It would be yes if, for example, we had measured the radius of gyration $\boldsymbol{R}_{\mathrm{g} \text { mesh }}$ of only one mesh labelled : for the usual molecular weight of a mesh in a rubber (3 000 to 100000 ), it is easy to measure it by SANS. Dependence of $R_{\mathrm{g} \text { mesh }}$ upon $\lambda$ is given for classical models such as the junction affine one (resp. the phantom network model), which leads to $R_{\mathrm{g}}(\lambda)=\left(\left(\lambda^{2}+1\right) R_{\mathrm{giso}} / 2\right)^{1 / 2}$ (resp. $\left.\left(\left(\lambda^{2}+3\right) R_{\text {giso }} / 4\right)^{1 / 2}\right)$. This has indeed been attempted $[4,5]$ for usual meshes, where $R_{\mathrm{g}}$ is small enough to have $q R_{\mathrm{g}} \ll 1$ in the available $q$ range. When one labels a path of several meshes $R_{\mathrm{g}}$ becomes a complicated function of $\lambda$ (tending to $R_{\mathrm{g}} \propto \lambda$ at very large $R_{\mathrm{g}}$ ), but it is still calculable. In our experiment, however the radius of gyration is so large that the condition $q<1 / R_{\mathrm{g}}$ is impossible inside the measurable range. This is also indeed an advantage : the form factor of a single chain will never give any information at a scale larger than $R_{\mathrm{g}}$; by having $q>1 / R_{\mathrm{g}}$ inside the whole measurable range, we exploit it at its best.

In order to compare experimental data and the form factors predicted by the models, we evaluated them numerically. First we will describe the data qualitatively.

4.2 REPRESENTATION OF $S(q)$. - To represent $S(q)$, we use the representation $q^{2} S(q)$ versus $q$ (Kratky plot). This leads to a plateau at high $q$ for a Gaussian chain $\left(q R_{\mathrm{g}}>5\right)$. A totally affine deformation (Eq. (2) at all scales) would also tend, at high $q$, to a plateau of ordinate $1 / \lambda_{q}^{2}$ where $\lambda_{q}$ is the macroscopic deformation in the direction of $q$ - in our case, $1 /(0.464)^{2}=$ 4.6 (we recall that $\lambda_{q}$ is the same for the gel and the melt).

Melt data. - Figure 1 shows the plot obtained for the smallest values of $t$. In the parallel direction, the curve increases continuously with $q$ up to the level of the isotropic sample; in the perpendicular direction we observe a maximum in the curve. A simple explanation for this is as follows. Over long distances, the conformation is very different from the isotropic one. 


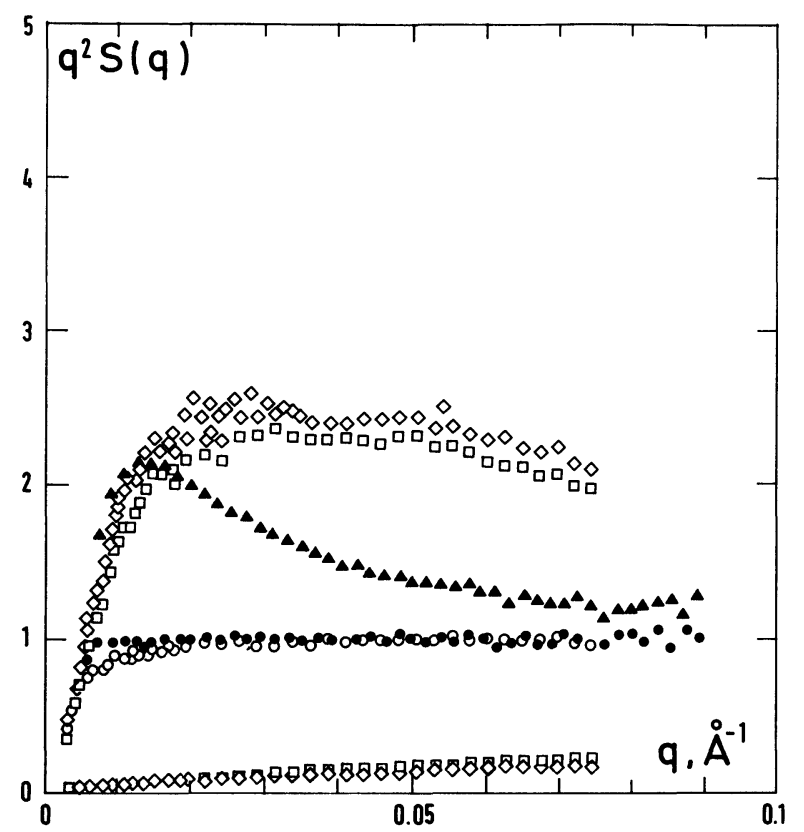

Fig. 1. - Kratky plot $\left(q^{2} S(q)\right.$ versus $\left.q\right)$ for measured single chain form factors : deswollen gel; - isotropic melt, measured in the same measurement period than the gel; $\diamond$ stretched melt, $t=60 \mathrm{~s}$ in both parallel (below) and perpendicular (above) direction; $\square$ stretched melt, $t=600 \mathrm{~s}$; $O$ isotropic melt, same measurement period as the stretched melts.

In the parallel direction the $q^{2} S(q)$ curve falls below the isotropic plateau, whereas in the perpendicular, it lies above it. At small distances the chain is no longer anisotropic, and $S(q)$ goes back to its isotropic values for both directions. In the perpendicular this leads to a maximum which attests to the loss of affineness. The curve for $t=10 \mathrm{~min},>t=1 \mathrm{~min}$, is slightly more relaxed (closer to the isotropic curve) than the curve for $t=1 \mathrm{~min}$. In contrast, the curve of $t=40 \mathrm{~min}$ (not shown here) remains equal, within the accuracy, to the one for $10 \mathrm{~min}$.

Gel data. - In figure 1, data for the dry gel are also shown. A good test of consistency with the experiment for stretched melts would be to compare the scattering from the two isotropic melts measured in each set of experiments. The superimposition appears satisfactory in this figure. For the dry gel, we observe a shape similar to that observed for melts in the perpendicular direction. We argue that the chain has been deformed on deswelling according to an isotropic compression similar to a perpendicular compression in uniaxial stretching. The difference between the dry gel and the isotropic Gaussian chains decreases as the distance decreases (i.e. as $q$ increases) and the hump attests similarly to this loss of affineness.

\section{Comparison with theories for the deswollen gel.}

The chain conformation in a strained elastomer is generally believed to depend on macroscopic defor- mation in a manner intermediate between the predictions of two theories $[6,7,17]:$ the so-called « junction affine" model and the "phantom " model. In the first one, the crosslinks are supposed to be fixed in space and their positions to be affinely transformed in the macroscopic dimensions of the sample. In the second, the junctions are assumed to fluctuate around affinely deformed mean positions. The difference between the two models arises from the hypothesis of independence on the strain of the fluctuations around the mean positions. The contribution of these fluctuations to the end-to-end vectors of the elementary chain is independent of the deformation. As a result, the affineness is partly lost at the scale of the mesh.

For the junction affine model, a general mathematical expression of the scattering function of a labelled path running through affinely displaced junctions was recently derived by $R$. Ullman [18]. A slightly simplified version of this derivation, but one adapted to the present case (high $q$ regime), is briefly reported in appendix 2 . The corresponding calculated curve (i.e. for a path of mass $2.6 \times 10^{6}$ linked to a deswollen network of mesh 35000 ) is plotted in the $q^{2} S(q)$ versus $q$ representation in figure 2 . It is immediately seen that this curve cannot fit our results. This corroborates the conclusion of several researchers $[19,20]$ who have already noticed that the "junction affine" model overestimates the chain deformation in loose networks.

The question is now to determine whether the smaller lower deformation we observe here can be accounted for by the fluctuations of the junctions

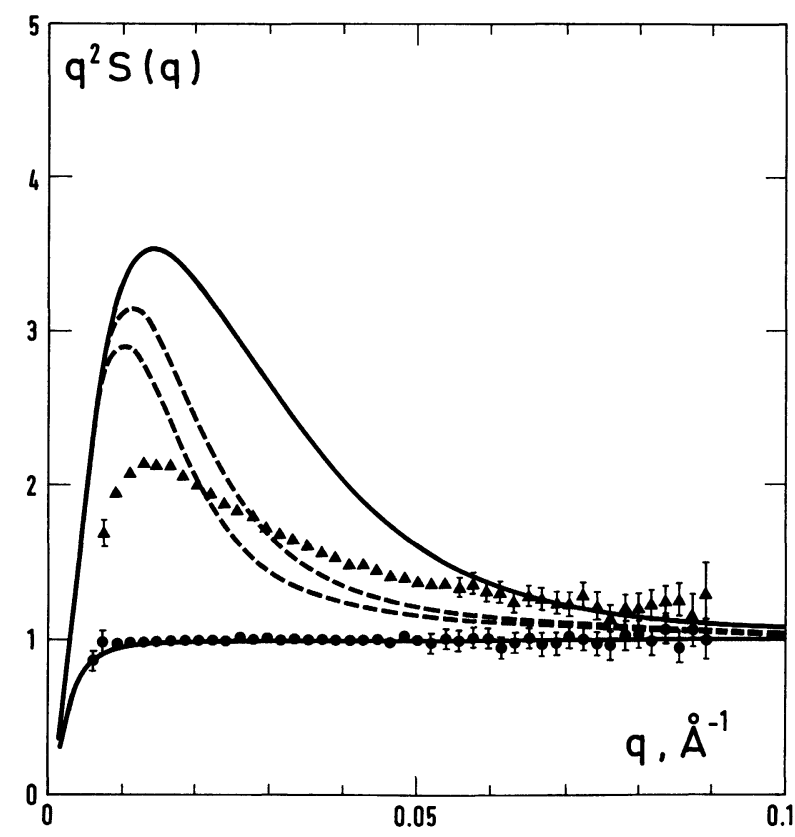

Fig. 2. - Comparison of gel data with calculated form factors, in a Kratky plot, for the same deformation ratio : isotropic Gaussian (plain line below) ; junction affine model, $M_{\text {mesh }}=35000$ (plain line above); phantom network model, $M_{\text {mesh }}=35000$ and $M_{\text {mesh }}=50000$ (below), dotted lines. 
assumed in the phantom model. The expression of the scattering function of a labelled path statistically linked to a " phantom network » has been calculated by Warner and Edwards. It is also given in appendix 2. The corresponding calculated curve obtained from the same parameters as in the preceding case ( $M_{\text {mesh }}$ now being an average value) is also plotted (dashed line) in figure 2. Though it is undeniably closer to the experimental points than the « junction affine " curve, the figures are not in good agreement at either low $q$ or at high $q$ values.

First we will discuss the low $q$ region. For $q \leqslant$ $2.5 \times 10^{-2} \AA^{-1}$, the experimental hump characteristic of the observed loss of affineness is far less pronounced than the phantom network curve. In other words, in the range of distances probed by the above scattering vector regime, the affineness is lost more rapidly than expected from any theory. However, this behaviour can be understood qualitatively as a manifestation of the rearrangement mechanism of neighbour junctions that was proposed recently [22].

Let us recall the basic idea. At the concentration of preparation, the average distance between crosslinks is roughly $110 \AA$, very comparable to the average endto-end distance of the estimated mesh $(125 \AA)$. Therefore most of the first neighbour crosslinks are connected by an elementary chain, and the gel can be visualized as a close packing of excluded meshes. After deswelling to the dry state, the average distance between the junctions is reduced to roughly $50 \AA$. Within the framework of the junction affine model, the average end-to-end distance of the mesh would be reduced to this value. Reference [22] proposes another way of escaping the constraints imposed macroscopically when the scale decreases. A network is a nearly one dimensional object filling space, and the relevant distance may not be the spatial one but the one along the chains as the latter themselves propagate the constraints. We may call it the " chemical » distance, and two junctions may be called first chemical neighbours if the shortest connection is one elementary chain, second chemical neighbours if it corresponds to two elementary chains, etc. Then, instead of the homogeneous folding of the junction affine model which keeps the spatial first neighbours, one can imagine a kind of tridimensional accordion-like folding which exchanges the spatial neighbours. After deswelling, some of the high order chemical neighbours may occupy spatially closer positions than first chemical neighbours, whose relative positions have been only slightly modified. In other words, a densityimposed average spatial distance between two first spatial neighbours of $50 \AA$ does not necessarily mean that these are first chemical neighbours. The spatial distance between two junctions reaches the affine value only if they are connected by a larger number of elementary chains.

We have not yet succeeded in making this mechanism quantitative for a prediction of path deforma- tion at different scales. However, two experiments can be explained by this model :

The first is the measurement of the radius of gyration of the mesh for different concentrations in osmotic deswelling of a gel (different from the deswelling concerned in this paper) [4]. When decreasing the concentration of the gel, starting from the equilibrium swelling value, the variation of the radius is nearly a plateau on a large range of concentrations. The mesh size appears very slightly deformed in this range.

The second is the measurement of the form factor of the elementary chain for uniaxially deformed PDMS rubbers with labelled meshes [5]. The relative deformation of the elementary chain decreases when its molecular weight $M$ increases, while the classical models predict no dependence on $M$.

Let us now discuss the discrepancy between experimental and theoretical curves at higher $q$ values $\left(q>2.5 \times 10^{-2} \AA^{-1}\right)$. The situation is different : the experimental curve crosses the phantom network prediction. The actual network seems therefore to be more deformed there than the phantom network. Since the typical range of distances probed by the experiment in this regime of $q$ is $10-40 \AA$, it can be understood that the capability of rearrangement begins to be reduced by uncompressibility. In this case it is not the fact that the mean positions of the junctions are affinely displayed which is in question, but rather the idea of chains with no volume (in other words chains of an axial diameter equal to zero). In short, the experimental result at low $q$ is indicative of a large freedom of the network at semilocal scale $\left(R_{\text {mesh }}<d<R_{\text {path }}\right)$, but the result at high $q$ shows that the freedom is weaker at lower scale, where the volume of the chain becomes relevant. The residual orientation is larger than predicted by any theory; this situation lasts when decreasing the scale down to the order of magnitude of the statistical unit. It is difficult to give quantitative results for that scale because of the low signal-to-noise ratio [23] at high $q$, but similar behaviours appear in other data. This can also be compared to results obtained from other techniques, such as fluorescent depolarization [24].

Remark. - Instead of fitting the different models for the real value of $Q$, the deswelling ratio, or of $\lambda$, the stretching ratio, we can, very simply, make $\lambda$ (or $Q$ ) vary in order to obtain a best fit of the models for $\lambda^{*}$ (or $Q^{*}$ ) (the concept of $\lambda^{*}$ has been used by several authors, as Ullman). Such fits will be discussed for gels as well as for melts in a following paper.

\section{Comparison with theory for melts.}

As we have shown in section 3 , the duration of relaxation of the melt samples obeys equations $(1 \mathrm{a}, \mathrm{b})$ and thus lies in the "plateau regime ". One can thus consider the melt as a transient network : consequently we will compare it first to the permanent network and second to the same expression of the form factor 
calculated for the crosslinked medium (with the appropriate mesh size) as it is indeed suggested by the current theoretical «tube » model.

6.1 Comparison with Deswollen gels. - The fulfilling of condition (1b) can be used first to compare results from deswollen gels and from melts with identical mesh size and deformation ratios directly. The molecular weight of the mesh " elementary chain " in the melt transient network is $\boldsymbol{M}_{\mathrm{e}}$, given as $\mathbf{2 0} 000$ for polystyrene [1]. The value for our gels, 35000 , is of the same order of magnitude. The perpendicular deformation for the melt is $1 / \sqrt{4.6}=0.466$; it can be compared to $1 / \sqrt[3]{10}=0.464$, the deformation ratio of the deswelling for gels. In figure 1, the curves are similarly shaped in both cases but if the figures are examined in detail, some differences appear. In the case of the melt, the ordinate of the peak is smaller and the "tail" at high $q$ is higher. It would be unrealistic to explain these differences by a difference in mesh size, which would in fact lead to the opposite effect. Other results in the same set of experiments on gels (to be published) indicate a decrease of the peak for a larger mesh size : gels with elementary chains of molecular weight 20000 would give a curve lying above the one corresponding to gels with larger mesh size $(M=35000)$, and thus even further away from the curve observed for melt.

6.2 Comparison with aVAilable models. - The old idea of the transient network has been used in different theories up to the most recent ones such as the " tube " theories using the de Gennes concept $[27,28]$ of reptation. In the Doï-Edwards model [29], most successful in describing the viscoelastic properties upon deformation, the relaxation at constant strain is described through three processes.

In the first process the chain moves as free for times smaller than $\tau_{\mathrm{tr}}^{\max }=T_{\text {Rouse }}\left(M_{\mathrm{e}}\right)$ - that is, for distances smaller than $D M_{\mathrm{e}}$. At larger times, the entangled medium is depicted as a fixed tube in which each chain moves. Consider a chain passing through some sliplinks. These are permanent crosslinks, but the chain can slip inside them as it « reptates " in the tube. The stress is calculated from that model using the classical theory of rubber elasticity, assuming the affine deformation of the mean positions of the junctions (i.e. the sliplinks). This leads to the second and the third processes. The second process is the equilibration of the chain inside the tube; we will return to it in the discussion. In the third process the chain disengages from the sliplinks at its two ends, so the number of sliplinks of decreases. Condition $(1 \mathrm{a}, \mathrm{b})$ allows to assume that the third process is not relevant here.

Following that picture, the tube model indeed sees the chain under $(1 \mathrm{a}, \mathrm{b})$ as a labelled path in a network. We could thus calculate the functions $S_{1}(q)$ in range (2) assuming the junction affine model with a mesh size corresponding to $M_{\mathrm{e}}=20000$. The calculated curve is presented in figure 3 : for the perpendicular direction the comparison of the data to the model shows a disagreement similar to that of the case of the deswollen gels with the same model (with $M=35000$ ). Using the phantom network calculation, we observe another bad agreement in figure 3 , although it is better than for the junction affine model. The use of both classical elasticity models inside the sliplink model thus leads to a disagreement with SANS measurements $\left({ }^{1}\right)$.

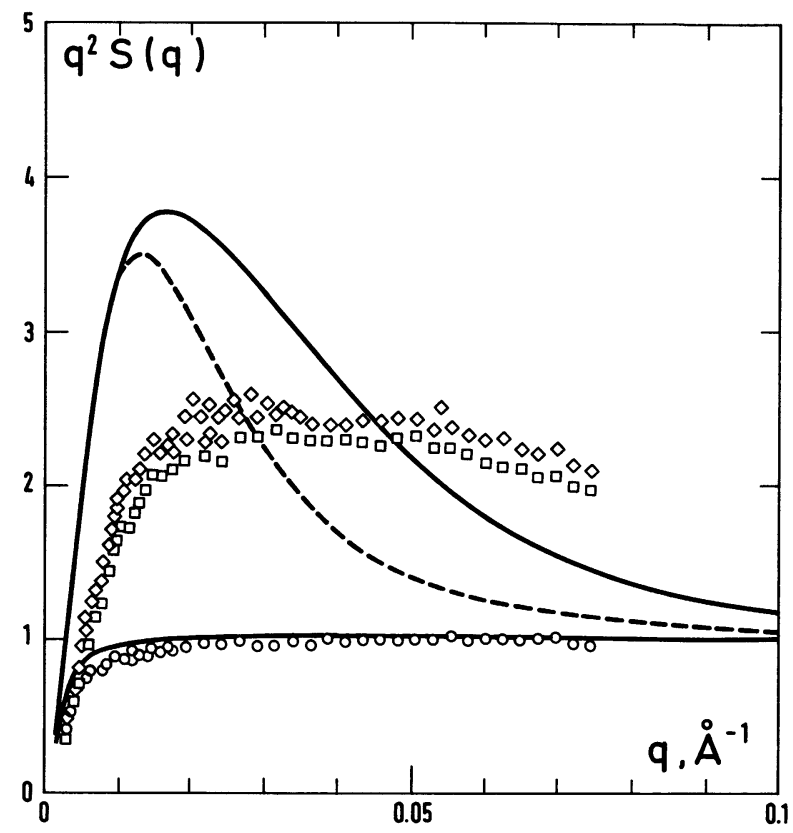

Fig. 3. - Comparison, for perpendicular direction, of stretched melt data with calculated form factors, in Kratky plot, for the same deformation ratio : isotropic Gaussian (plain line below); junction affine model, $M_{\text {mesh }}=20000$ (plain line above); phantom network model, $\boldsymbol{M}_{\text {mesh }}=35000$ (dotted lines).

6.3 Discussion. - The classical elasticity models do not quite describe the $q R_{\mathrm{g}}>1$ regime for the strained melt in the plateau regime as well as for deswollen gels which are strained rubbers. Though there are some unforeseen discrepancies in the direct

( $\left.{ }^{1}\right)$ We used a cruder model, previously $[9,10]$, one that we call a sliding mesh model, and it is described in appendix 2 (Sect. A.3).

The comparison with experimental data on melts shows a larger disagreement than for either the junction affine or the phantom models. In a previous work $[9,10]$, we compared data using the sliding mesh model in parallel as well as in perpendicular directions. The agreement was much better in the parallel direction than we observed here. In the perpendicular direction it was less satisfactory but better. This might be due to the fact that the elongation is here 4.6, while it was only 3 in the previous work. This will be discussed elsewhere [30]. 
comparison of data for the two materials, we are tempted to keep the idea of a similarity between these two deformed networks and to point out the weakness of the theoretical treatment of such a network. A theory giving predictions closer to our experimental SANS results could be used to hypothesize more about the dynamics of melt in particular. It may also help develop ideas about reptation theories such as Doi and Edwards' theory.

At this point we would like to return to the second process, that of equilibration. At this stage, in the fixed tube, the linear density along the centre line can be calculated. It appears that it has a lower value than any found in the isotropic melt because of the geometrical properties of an affinely deformed path in more than one dimension. The return to equilibrium density via slipping in the links (or the tube) corresponds to a contraction. This model allows us to explain an overstress in high deformation cases and a decrease in stress in a time proportional to $M^{2}$ [31-33]. This contraction should also lead to a decrease in the radius of gyration in the same time; however, our detailed measurement of that radius $[8,9,10,40]$ failed to observe the contraction. We can now propose an explanation for this failure : it could be possible if the tube is no longer completely fixed. The motion of the tube would be parallel to the reptation processes; and it has been proposed that this would be due to the motions of the other chains which would destroy some of the entanglements (" tube renewal "). However, we propose that the entanglements are still permanent (so that there would be no difference with a crosslinked melt), but that the tube moves via some " rearrangements ", escaping the affine deformation of the tube. This would explain the smoothed and widened loss of affineness observed here, and possibly correspond to the interpenetration effect discussed in section 5 as well.

\section{Conclusion.}

Let us summarize the results we put forth in this paper. In a deswollen gel, the deformation of the chain on a semilocal scale presents a loss of affineness that does not correspond to the predictions of the classical theories. It is interpreted as a manifestation of a nonaffine reorganization of junction positions. In a range of times sufficiently small compared to characteristic time associated with terminal processes, a deformed melt is believed (in reptation theories, for example) to behave as a network of temporary entanglements. Such a melt should also escape the affineness in the macroscopic strain by the rearrangement of temporary junctions. In fact, the experimental curves for the melt cannot be fitted by using the classical theoretical calculations, whereas they are more comparable to those of permanent networks. The dynamics of this reorganization mechanism could play an important role in the relaxation process of melts. Moreover, this particular loss of affineness could lead to an equilibrium of the linear density of the chain without the help of the unobserved contraction along the tube.

Finally, we insist on the fact that the data interpreted here are preliminary; we recently obtained better data by comparing a stretched melt directly with a stretched crosslinked melt with the same deformation history. Representative results, which tend to support these findings, are given in reference [30] and they will be published in detail.

\section{Acknowledgments.}

We are deeply indebted for experimental collaboration and discussions to Kunihiro Osaki (especially about the "contraction ") and Martine Nierlich. We had many enlightening discussions with Ludwik Leibler. We thank Claude Cohen a lot for his helpful comments on the manuscript. We also wish to express our appreciation to Dr. G. Beinert for his pertinent technical advice and to Mr. F. Isel for his very efficient technical assistance.

\section{Appendix 1.}

DISCUSSION OF THE SYSTEMATIC ERROR ASSOCIATED WITH THE DIFFERENCE BETWEEN THE MOLECULAR WEIGHT OF THE LABELLED CHAIN AND THAT OF THE MATRIX IN NEUTRON SCATTERING EXPERIMENTS. In this paper, unless the molecular weight of the matrix and deuterated chains are different $\left(N_{\mathrm{H}} \neq N_{\mathrm{D}}\right)$, we have postulated the use of equation (4) instead of (3). This is right only for $S_{\mathrm{D}}(q)=S_{\mathrm{H}}(q)$. Doing that, we therefore committed a systematic error

$$
\varepsilon(q) \sim \phi_{\mathrm{D}}\left(1-N_{\mathrm{D}} P_{\mathrm{D}}(q) / N_{\mathrm{H}} P_{\mathrm{H}}(q)\right) .
$$

We want in this appendix to estimate the order of magnitude of this error in the different cases we considered.

(i) The gel. - In addition to considering the case of very large deuterated chains A $\left(M=2.6 \times 10^{6}\right)$, we performed the same experiment (deswelling. of a network by a factor of 10) on deuterated paths B of molecular weight close to that of the undeuterated chains $\left(M=8 \times 10^{5}\right)$. The results concerning this type of paths will be discussed in greater detail elsewhere [39]. We shall use only the crude data here in order to estimate $N_{\mathrm{H}} P_{\mathrm{H}}(q)$ in the case of paths A which is very close to $N_{\mathrm{H}} P_{\mathrm{H}}(q)$ for paths $\mathrm{B}$. Since the molecular weight of the matrix chains and the paths $B$ are rather close to each other, we can write :

$$
N_{\mathrm{D}}^{\mathrm{B}} P_{\mathrm{D}}^{\mathrm{B}}(q)=S^{\mathrm{B}}(q) /\left(1-\phi_{\mathrm{D}}\right) \phi_{\mathrm{D}}=N_{\mathrm{H}} P_{\mathrm{H}}(q)
$$


where $N_{\mathrm{D}}^{\mathrm{B}} P_{\mathrm{D}}^{\mathrm{B}}(q)\left(\right.$ resp. $\left.N_{\mathrm{H}} P_{\mathrm{H}}(q)\right)$ represents the scattering function of the path $B$ (resp. of the matrix chain) and $S^{\mathbf{B}}(q)$ the normalized coherent cross-section scattered by the mixture. Therefore the normalized cross-section scattered by path $B$ gives us an estimation of the scattering function $N_{\mathrm{H}} P_{\mathrm{H}}(q)$ of the matrix chains for both A and B.

In figure 4 we have plotted in a Kratky plot representation $N_{\mathrm{D}}^{\mathrm{A}} P_{\mathrm{D}}^{\mathrm{A}}(q)$ and $N_{\mathrm{D}}^{\mathrm{B}} P_{\mathrm{D}}^{\mathrm{B}}(q)=N_{\mathrm{H}} P_{\mathrm{H}}(q)$. The ratios of the corresponding ordinates of the curves will lead to an estimation of $\delta=N_{\mathrm{D}}^{\mathrm{A}} P_{\mathrm{D}}^{\mathrm{A}}(q) / N_{\mathrm{H}} P_{\mathrm{H}}(q)$. At the very beginning of the curves $\delta$ is of the order of 2 , and then, in the asymptotic regime, it decreases to 1.2 or even less. We can conclude that $\varepsilon(q)$ will be nearly one percent at the beginning of the curve and even less at larger $q$. Consequently $\varepsilon$ is, in this case, completely negligible.

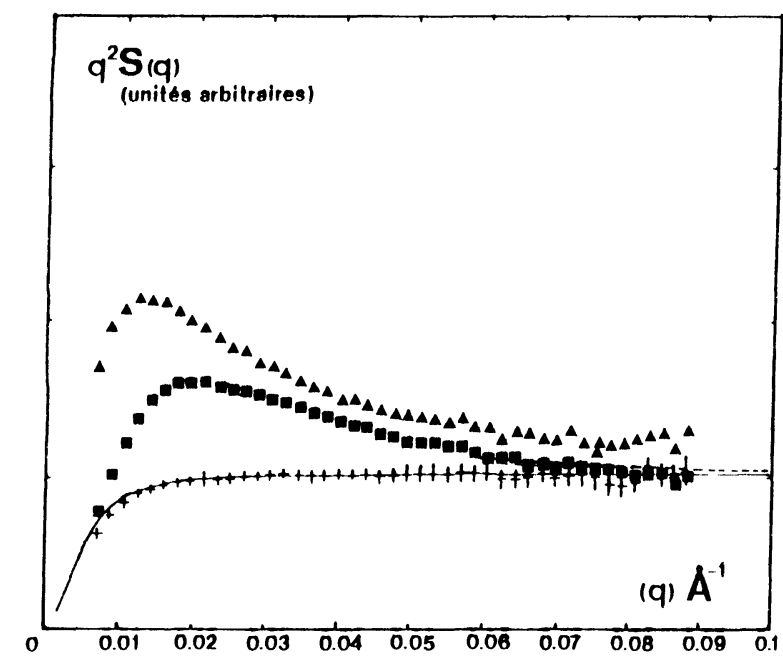

Fig. 4. - Comparison, in Kratky plot, between the form factors of two different labelled paths in matrices crosslinked and deswollen under the same conditions (deswelling ratio of 10). $\triangle$ Molecular weight of the labelled path $M_{\mathrm{w}} \cong 2.5 \times$ $10^{6}$ (same data as in Figs. 1 and 2, same symbols). $\square$ Shorter path $\left(\cong 8 \times 10^{5}\right)$.

(ii) The melt. - In the case of the melt, we did not measure the intensity scattered by a chain of same length as that of the matrix. Nevertheless, since the polymerization index is closer in the case of the melts (by roughly a factor of 2 ), it can be reasonably postulated that $\delta$ will be closer to unity, ranging between 1.5 in the small $q$ regime and 1.2 in the high $q$ regime. $\varepsilon(q)$ will therefore be of the following orders of magnitude :

$$
\begin{array}{lll}
q \cong 1 & \text { to } 2 \times 10^{-2} \AA^{-1} & \varepsilon \cong 0.05 \\
q \cong 6 & \text { to } 8 \times 10^{-2} \AA^{-1} & \varepsilon \cong 0.01
\end{array}
$$

We observe that this systematic error $\varepsilon$ is not important enough to modify the conclusions of this study in the smaller $q$ regime, and it is negligible in the larger $q$ regime.

\section{Appendix 2.}

CALCULATION OF THE INTENSITY SCATTERED BY DEFORMED LABELLED PATHS. - The scattering of a deformed Gaussian chain has been studied in several works. To interpret neutron scattering experiments, very simple predictions were made some time ago [35]. Calculations were done for chains stretched by their ends only. The " Gaussian approximation " for scattering, which just needs the mean square value $\left\langle r_{i j}^{2}\right\rangle$ $\left(\mathbf{r}_{i j}\right.$ vector between monomers $i$ and $j$ ), was used. For the classical model in which the ends were affinely displaced, one used the Katchalski [36] expression of $r_{i j}^{2}$. For the model in which the ends were the crosslinks of a phantom network, one obtained an expression of $r_{i j}^{2}$ very close to Katchalsky's [37]. For a labelled path, i.e. a chain deformed by several points, only the simple sliding mesh model was calculated [38].

A.1 Affine deformation of the junctions. - The method we use is that developed by R. Ullman [18]. Unfortunately this author did not consider the " intermediate " regime $\left(q R_{\mathrm{g}} \gg 1\right)$, which is of interest here. We had, therefore to make the calculation again. It can be notably simplified by taking into account the large number (greater than 50) of elementary meshes involved in the labelled paths. Thus the fraction of scattering centres located on the loose ends is low, and the contribution of the correlation between them or with other points of the chain is weak. It is therefore reasonable to perform the calculation as if the chains were terminated by crosslinks.

A second approximation can be made by assuming the paths to be divided in a succession of regular elementary meshes. Actually, because of the statistical linking of the chains, the lengths of the sequences will be distributed around their average value. This distribution (which is not known) should be taken into account; however, we are not convinced that this would lead to an improvement in handling the physics of the problem. The assumption of affine deformation of the crosslink positions, while crude for a regular network, is far more difficult to admit in the case of irregular meshes. Taking into account the whole distribution should not introduce more than a șlight change at high $q$ values. Since we observe a more fundamental disagreement with experimental data (in the whole domain of the scattering vector), such an improvement would be useless. We simply examined, at first, in this paper, the following question : is the deformation of a real network of average mesh $M$ and average functionality $f$ comparable to the deformation of an ideal network of monodisperse mesh $M$ and functionality $f$ whose junction positions are affinely deformed ? We performed the calculation again, using a slightly simplified approximation. We have considered a path beginning and ending at a crosslink that is, we neglected the loose ends that exist in the case of statistical linking. Since the molecular weight of the path $\left(M_{\mathrm{n}} \sim 2.6 \times 10^{6}\right)$ is very large compared to 
that of the mesh, the proportion of dangling segments is weak $\left(\sim 2 \times 10^{-2}\right)$ and can be neglected.

The intrachain correlation function of a labelled path can be expressed as

$$
\begin{aligned}
S(q) & =\frac{1}{N^{2}} \sum_{i} \sum_{j}\left\langle\mathrm{e}^{i \mathbf{q} \cdot \mathbf{r}_{i j}}\right\rangle \\
& =\frac{1}{(n p)^{2}} \sum_{\alpha=1}^{n} \sum_{i_{\alpha}=1}^{p} \sum_{\beta=1}^{n} \sum_{j_{\beta}=1}^{p}\left\langle\mathrm{e}^{i q\left(\mathbf{r}_{i_{x}(\alpha+1)}+\mathbf{r}_{(\alpha+1) \beta}+\mathbf{r}_{\beta j_{\beta}}\right)}\right\rangle
\end{aligned}
$$

where $N$ is the number of scattering centres on the path and

- $n$ the number of subunits along the path corresponding to elementary chains (meshes),

$-p$ the number of diffraction centres in a subunit,

- Greek letters index the junctions, and

$-i_{\alpha}, j_{\beta}$ index the centres $i$ and $j$ in the subchains beginning at $\alpha$ and $\beta$ respectively.

In the case of affine deformation of junction positions, the vectors $\mathbf{r}_{i_{\alpha}(\alpha+1)}, \mathbf{r}_{\alpha+1 \beta}, \mathbf{r}_{\beta j_{\beta}}$ are independent variables, so (A .1) becomes the following :

$$
S(q)=\frac{1}{(n p)^{2}}\left[n \sum_{i=1}^{p} \sum_{j=1}^{p}\left\langle\mathrm{e}^{i \mathbf{q} \cdot \mathbf{r}_{i j}}\right\rangle+\sum_{i_{\alpha}=1}^{p}\left\langle\mathrm{e}^{i \mathbf{q} \cdot \mathbf{r}_{i \alpha(\alpha+1)}}\right\rangle \sum_{j_{\beta}=1}^{p}\left\langle\mathrm{e}^{i \mathbf{q} \cdot \mathbf{r}_{\beta j_{\beta}}}\right\rangle \sum_{\alpha=1}^{n} \sum_{\substack{\beta=1 \\ \alpha \neq \beta}}^{n}\left\langle\mathrm{e}^{i \mathbf{q} \cdot \mathbf{r}_{\alpha+1}+1_{\beta}}\right\rangle\right]
$$

where

$$
A(q)=\sum_{i=1}^{p} \sum_{j=1}^{p}\left\langle\mathrm{e}^{i \boldsymbol{q} \cdot \mathbf{r}_{i j}}\right\rangle, \quad B(q)=\sum_{i_{\alpha}=1}^{p}\left\langle\mathrm{e}^{i q \cdot \mathbf{r}_{i_{\alpha}(\alpha+1)}}\right\rangle, \quad B^{\prime}(q)=\sum_{j_{\beta}=1}^{p}\left\langle\mathrm{e}^{i \mathbf{q} . \mathbf{r}_{\beta_{j \beta}}}\right\rangle
$$

correspond to intrasubchain correlations. In the case of affine deformation of the junctions, these functions are given by the Katchalsky expression [36], which reads in Ullman's notation as

$$
\begin{gathered}
A(q)=p+2 p \sum_{u=1}^{p}\left(1-\frac{u}{p}\right) \exp \left[-q^{2} \frac{p a^{2}}{6}\left(\frac{u}{p}+\left(\frac{u}{p}\right)^{2}\left(\lambda^{2}-1\right)\right)\right] \\
B(q)=B^{\prime}(q)=\sum_{v=1}^{p} \exp \left[-q^{2} \frac{p a^{2}}{6}\left(\frac{v}{p}+\left(\frac{v}{p}\right)^{2}\left(\lambda^{2}-1\right)\right)\right]
\end{gathered}
$$

In the case of an isotropic deformation, $\left(\frac{v}{v_{0}}\right)=\lambda^{3}$, and the modulus of $\mathbf{q}$ is involved. The remaining function $C(q)=\sum_{\alpha=1}^{n} \sum_{\beta=1}^{n}\left\langle\mathrm{e}^{i \mathbf{q} \cdot \mathbf{r}_{\alpha+1 \beta}}\right\rangle$ is easy to evaluate. The assumption of affine deformation of crosslink points results in a Gaussian form for the distribution function of vectors $\mathbf{r}_{\delta \varepsilon}$ connecting them. Only the second moments are changed in

$$
\left\langle r_{\delta \varepsilon}^{2}\right\rangle=|\delta-\varepsilon| \lambda^{2} p a^{2} .
$$

Then

$$
\begin{aligned}
C(q)=2(n-1)+2 & \sum_{s=1}^{n-2}[(n-1)-s] \times \\
& \times \exp \left(-q^{2} s \lambda^{2} \frac{p a^{2}}{6}\right) .
\end{aligned}
$$

The series $A(q), B(q)$, and $C(q)$ are easily calculated using a desk top computer. In practical terms, we have taken a spacing of the scattering centres corresponding to a molecular weight of 100 . In the undeformed state $(\lambda=1)$ with the $q^{2} S(q)$ representation, we obtain the plateau shape, typical of the Gaussian character of the chains. As seen in figures 2 and 3 , it is a good fit of the experimental undeformed melt. For the deformed state, we give three calculated curves in figure 5 corresponding to the same labelled mesh $\left(M=2.6 \times 10^{6}\right)$ and differing by the lengths of the subunit $\left(M_{\text {mesh }}=20000,35000,50000\right)$, which can be compared to the isotropic curve for the same molecular weight.

A. 2 Phantom network model. - In the so-called phantom network model, only the mean positions of the junctions are affinely transformed in the macroscopic strain. The junctions are assumed to fluctuate around their mean positions independently of the state of strain. As a result, the instantaneous values of the end-to-end vectors are not transformed affinely. Theoretical expressions of the intensity scattered by labelled paths in the phantom network have been given by Warner and Edwards [21] and Ullman [18]. We have performed numerical evaluations for both of them. A slight difference between the systems has been observed, and we attribute it to an approximation in Ullmann's formulation : a vector $\mathbf{r}_{i j}$ connecting monomers is written as a sum of three vectors which are considered as independent variables (in the same manner as in the preceding paragraph before equa- 


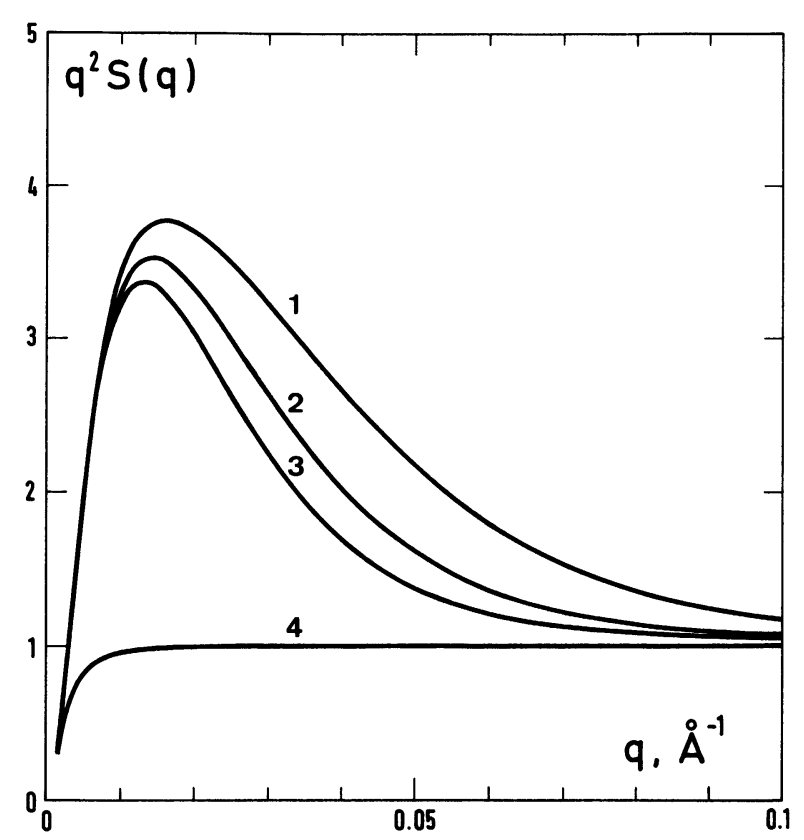

Fig. 5. - Kratky plot for calculated form factor for the junction affine model, with a deformation ratio of 0.464 . Curve 1: $M_{\text {mesh }}=20000 ;$ curve $2: M_{\text {mesh }}=35000$; curve 3 : $M_{\text {mesh }}=50000$; curve 4 : isotropic Gaussian.

tion (A.3)). The correlation of the fluctuations of neighbour junctions in the phantom model contradicts this assumption of independence and introduces a slight underestimation of the deformation. The curves we report below are numerical evaluations of the expression obtained by Warner and Edwards using a direct, elegant application of the replica method.

Within our notations, this expression has the following form :

$$
\begin{aligned}
& S(q)= \\
& \begin{aligned}
\frac{1}{N^{2}} \sum_{\substack{i \\
\text { path }}} & \exp \left\{-\frac{q^{2} a^{2}}{6}\left[\lambda^{2}|i-j|+p\left(1-\lambda^{2}\right)\right.\right. \\
\times & {\left.\left.\left[1-\exp -\frac{(i-j)}{p}\right]\right]\right\} . }
\end{aligned}
\end{aligned}
$$

The first term in the exponential corresponds to a chain affinely deformed at any scale, while the second represents the non-affine fluctuation that the chain executes. The curves which are plotted in figure 6 within the Kratky Porod representation have been obtained for three values of the elementary mesh of the network, corresponding to molecular weights of 50000,35000 , and 20000 . The sums have been changed to integrals and calculated using the Simpson method.

A.3 Sliding mesh model. - In addition to the two preceding models we can also consider a different calculation, one which ignores the fact that the junctions have definite positions on the chains by intro-

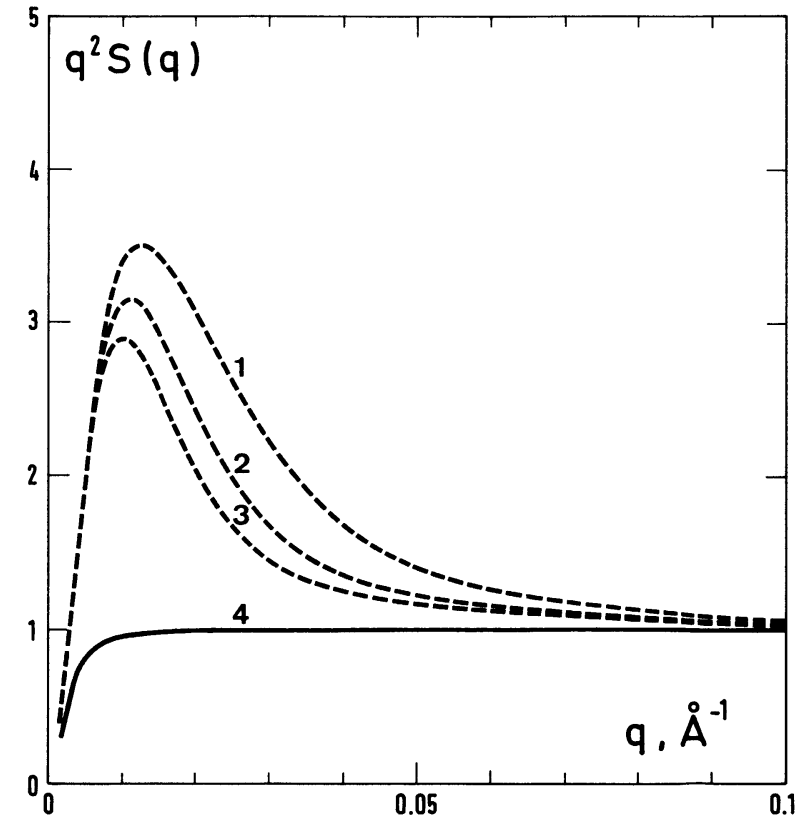

Fig. 6. - Kratky plot for calculated form factor for the phantom network model, with a deformation ratio of 0.464 . Curve 1: $M_{\text {mesh }}=20000 ;$ curve $2: M_{\text {mesh }}=35000$; curve 3: $M_{\text {mesh }}=50000 ;$ curve $4:$ isotropic Gaussian.

ducing only a cut-off of the affiness in terms of chemical distance along the chain between two points. We will see that it illustrates the role of the fluctuations of the chains in networks especially well.

Let us suppose that for any pair of points located at distances along the path larger than a certain critical

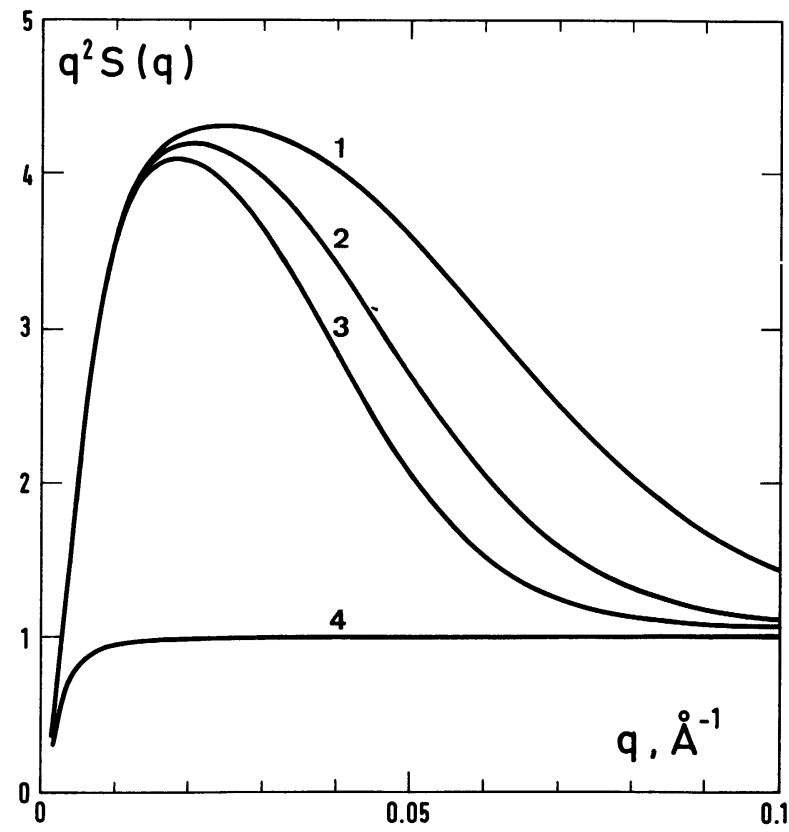

Fig. 7. - Kratky plot for calculated form factor for the sliding blob model, with a deformation ratio of 0.464 . Curve $1: M_{\text {blob }}=20000$; curve $2: M_{\text {blob }}=35000$; curve 3 : $M_{\text {blob }}=50000 ;$ curve $4:$ isotropic Gaussian. 
threshold $l$, the deformation is affine in the macroscopic strain

$$
\begin{array}{r}
\left\langle r_{i j}^{2}\right\rangle=\lambda^{2}\left\langle r_{i j}^{2}\right\rangle_{0}=\lambda^{2}|i-j| a^{2} \\
\text { for }|i-j|>l .
\end{array}
$$

On the other hand, at distances shorter than $l$ the affineness is progressively lost. We need an expression for that loss of affineness, and we take the simplest, the Katchalsky function given in equation (A.3). Within the framework of these assumptions the scattering function of the path reads as follows :

$$
\begin{aligned}
& S(q)=2 \sum_{v=1}^{l}(N-v) \times \\
& \quad \times \exp \left\{-q^{2} \frac{N a^{2}}{6} \times \frac{l}{N}\left[\left(\frac{v}{l}\right)+\left(\frac{v}{l}\right)^{2}\left(\lambda^{2}-1\right)\right]\right\}
\end{aligned}
$$

$$
\begin{aligned}
& +2 \sum_{v=l+1}^{N-1}(N-v) \exp \left(-q^{2} \lambda^{2} \frac{v a^{2}}{6}\right) \\
& =F(q)+G(q) .
\end{aligned}
$$

The first sum is calculated using the same method as $A(q)$, while the second is easily integrated. $S(q)$ has been calculated for three values of $l$ corresponding to the preceding meshes of 20000,35000 and 50000 . The results are reported in figure 7 . The humps are much more pronounced here than in the junction affine model : the deformation is larger. This arises from the fact that when the junctions are affinely deformed, the chains partly relax between them, and this effect also diminishes the deformation for pairs which are not located on the same subunits. This effect is cancelled for $|i-j|>l$ in the sliding mesh model.

\section{References}

[1] FERRY, J. D., Viscoelastic properties of polymers (J. Wiley, New York), 3rd ed., 1983.

[2] Isotropic conformation was first measured using a small concentration $\varphi_{\mathrm{D}}$ of labelled chains. CoTTON, J. P. et al., Macromolecules 7 (1974) 863. It was then shown to be possible to use any concentration $\varphi_{\mathrm{D}}$ for isotropic melts as well as anisotropic melts in reference [3].

[3] Boué, F., Nierlich, M., Leibler, L., Polymer 23 (1982) 29.

[4] Bastide, J., Duplessix, R., Picot, C., Candau, S., Macromolecules, January 1984.

[5] Beltzung, M., Picot, C., Herz, J., Macromolecules, December 1983.

[6] Flory, P. J., Proc. R. Soc. A 351 (1976) 1666.

[7] Deam, R. T., Edwards, S. F., Philos. Trans. R. Soc. A 280 (1976) 317.

[8] Boué, F., Nierlich, M., Jannink, G., Ball, R. C., J. Physique 43 (1982) 137.

Boué, F., Nierlich, M., Jannink, G., Ball, R. C., J. Physique Lettres 43 (1982) L-585, L-593.

[9] BouÉ, F., Thèse d'Etat, University of Paris-Sud Orsay, $n^{0} 2662$ (1982).

[10] Boué, F., Nierlich, M., Osaki, K., J. Chem. Soc. Faraday Trans. 18 (1983).

[11] Farnoux, B., Daoud, M., Cotton, J. P., Jannink, G., NiERLICH, M. and BoUÉ, F., J. Appl. Crystallogr. 11 (1978) 511.

[12] More clearly, see DE GenNES, P. G., Scaling concepts in polymer physics (Cornell University Press, Ithaca) 1977, chap. V, $\S 3.4$, we are here in the case $R_{\mathrm{B}}>\xi$.

[13] Schosseler, F., Bastide, J., Marchal, J., to be published.

[14] Dusek, K., Prins, W., Adv. Polym. Sci. 6 (1969) 1.

[15] Munch, J. P., Candau, S., Herz, J., Hild, G., J. Physique 38 (1977) 971.

Munch, J. P., Lemaréchal, P., Candau, J., Herz, J., J. Physique 38 (1977) 1439.

[16] Beltzung, M., Picot, C., RempP, P., Herz, J., Macromolecules 15 (1982) 1594

[17] Flory, P. J., J. Chem. Phys. 66 (1977) 5720.
[18] Ullman, R., Macromolecules 15 (1982) 1395.

[19] Clough, S. G., Maconnachie, A., Allen, G., Macromolecules 13 (1980) 774.

[20] BeltzUnG, M., Thesis, Strasbourg (1982).

[21] Warner, M., Edwards, S. F., J. Phys. A 11 (1978) 1649.

[22] Bastide, J., Picot, C., Candau, S., J. Macromol. Sci. Phys. B 19 (1980) 13.

[23] In more appropriate words the coherent part of the signal is small compared to the incoherent part and thus becomes of the same order as the dispersion if too short counting times are used.

[24] Monnerie, L., J. Chem. Soc. Faraday Trans. 18 (1983).

[25] Tовоlsky, A. V., J. Appl. Phys. 27 (1956) 673.

[26] Williams, M. L., Landel, R. F., Ferry, J. D., J. Am. Chem. Soc. 77 (1955) 3701.

[27] De Gennes, P. G., J. Chem. Phys. 55 (1971) 572.

[28] De Gennes, P. G., Léger, L., Ann. Rev. Phys. Chem. 33 (1982) 49-61.

[29] Doï, M., Edwards, S. F., J. Chem. Soc. Faraday Trans. 274 (1978) 1802.

[30] BouÉ, F., submitted to Adv. Polym. Sci.

[31] Osaki, K., Kurata, M., Macromolecules 13 (1980) 671.

[32] Osaki, K., Kimura, S., Kurata, M., J. Polym. Sci. Polym. Phys. Ed. 19 (1981) 517.

[33] OsaKi, K. et al., Macromolecules 14 (1981) 455.

[34] BouÉ, F., OSAKI, K., NierLich, M., to be published. This paper contains results for large molecular weight $\left(2.6 \times 10^{6}\right)$ confirming the results for smaller molecular weight $\left(7 \times 10^{5}\right)$ of reference [6].

[35] Benoit, H., Duplessix, R., Ober, R., Daoud, M., Cotton, J. P., Farnoux, B. and Jannink, G., Macromolécules 8 (1975) 451.

[36] Kuhn, W., Künzle, O. and Katchalsky, A., Helv. Chim. Acta XXXI, Fasc. VII (1948) 1994-2036.

[37] Pearson, D. S., Macromolécules 10 (1977) 696-701.

[38] Bout́, F., Jannink, G., J. Physique Colloq. 37 (1978) C2-183.

[39] BASTIDE, J., Thèse d'Etat, Université Louis Pasteur, Strasbourg (1985). 